\title{
Editorial
}

\section{Novel targets for CNS gene therapy}

The mammalian central nervous system (CNS) is the most complex of all somatic organs having a high degree of specialization and localization of function, extensive networking among cellular constituents and markedly limited capacity for regeneration. While this complexity precludes the facile application of rational, disease mechanism-based gene therapies it also provides a unique substrate that can be exploited to enhance the effectiveness of transduced genes. Herein, I will expand on this notion by discussion of specific neurologic diseases as novel targets for gene therapy.

The epilepsies constitute a group of neurologic disorders characterized clinically by various seizure syndromes and cellularly by hyperexcitability. The cell biologic problem is that of pathologic shifting of the normally well-regulated balance between inhibition and excitation towards the latter. Most acquired epilepsy is without definite etiology although an emerging viewpoint is that antecedent, perhaps subclinical events contribute to or trigger the development of the neural substrate underlying the hyperexcitable state (reviewed in Ref. 1). Since the processes underlying epileptogenesis are not fully elucidated, the treatment strategies are therefore directed at controlling symptoms (seizures). The seizures are the clinical manifestations of aberrant hypersynchronization of neural networks that can remain local, spread to other locations and/or involve all cortical regions at once. Neocortical or hippocampal circuits are uniformly activated during an epileptic seizure. Synaptically connected regions may be recruited in a manner to augment or attenuate the discharge. When epileptic discharges travel unimpeded through neural networks, the network functions are subverted, temporarily rendering them incapable of executing normal tasks. With increasing duration of disease, particularly in epilepsies involving the temporal lobe, the neural substrate scars, presumably from chronic exposure to excitatory neurotransmitters (eg mesial temporal sclerosis).

Among the most vexing of epilepsies are those refractory to medical therapy; patients with intractable seizures - usually, partial seizures - often come to evaluation for prospective neurosurgical resection. Notwithstanding the apparent large capacity of the primate brain we recognize that some regions are quite eloquent, demanding that any contemplated surgery be approached with the greatest caution. Such medically unresponsive and surgically difficult cases may prove suitable targets for gene therapy. The choice of genes to deliver would be based on the pathophysiologic sub- strates. Depending on the particular subtype and duration of disease these could represent simply excessive excitation or altered neural circuitry. Among potential gene candidates to consider are those anticipated to reduce the depolarization or synchronization of a focus or its subsequent spreading to downstream networks. ${ }^{2,3}$ By taking advantage of the apparent requirement for neural networks to transmit hyperexcitability it is possible to envision a gene therapy that can act locally to disrupt this process. Myriad considerations abound: Which cell types are to be targeted? Will the gene product act locally, diffusely or perhaps transsynaptically? Will constitutive expression preclude normal neurotransmission? Will disruption of unaffected neighboring circuitry result in loss of activity-dependent plasticity? Can the therapy be shut-down when symptoms abate? The ideal approach would naturally restrain focus bursting and activation of linked pathways without producing alteration in functional properties of adjacent networks. Like all preclinical gene therapy, the adequacy and relevance of models will be at issue. The choice of good models, recognition of limitations and translational extension all need to be considered (see Ref. 4). Although as yet undeveloped as a gene therapy target it would be wise to concurrently design and evaluate vectors capable of transduction of the desired substrate..$^{5-7}$

Arteriovenous malformations (AVMs) represent another potentially novel target for CNS gene therapy. These lesions, reflecting dysgenesis of the vasculature, have the proclivity to bleed and produce morbidity (including seizures) and death. Current approaches to AVMs include embolization, isolation and resection and radiation-induced sclerosis. The choice of modality is predicated on location, accessibility to stereotactic instrumentation and definition of vascular anatomy. Development of gene therapeutic strategies for AVM poses an interesting challenge. Considering the goal being to prevent hemorrhage one can devise approaches whereby endovascular gene delivery may lead to sufficient transduction to produce bioactive proteins within the vascular wall. These genes could be those encoding proteins with locally active thrombotic effects. Whereas unregulated expression of thrombus inducing or other lumen obliterating genes would be deleterious if widespread one can envision focal, AVM-specific, gene regulation using targeted radiation. Such a bimodal therapeutic design could theoretically allow for lower radiation burdens because radiation inducible promoters can be activated at smaller doses than is generally given when a primary therapy. ${ }^{8}$ Success with radiation inducible preclinical gene therapy for neoplastic disease supports the application of this 
approach to AVM. ${ }^{9}$ Although technical issues relating to the adequacy of gene transfer to the endothelium of the AVM will be formidable, existing methods for vessel microcatheterization may be applicable with modification. ${ }^{10}$

In many CNS diseases, neuronal loss is a prominent feature; the resultant neurologic dysfunction is permanent and often disabling. To combat disease-associated deleterious stimuli the post-mitotic neuron is endowed with multiple defensive mechanisms, some cell autonomous and others dependent on neighboring or networked cells. In numerous neurodegenerative diseases there is an inexorable progression from cellular dysfunction to death. The concept that early disease may reflect loss of function rather than cell death invokes the attractive and well-considered neuroprotective approach. However, unlike conventional, albeit rationally designed drugs, genes can be selectively targeted to afford protection where most desired, thus potentially averting unwelcome side-effects. One additional capability that may be uniquely within the purview of gene therapy is embodied in the idea of pathophysiologically entrained therapeusis. Simply formulated, a particular disease mechanism produces cellular perturbations in an episodic and unpredictable manner. Once characterized, the disease mechanism itself could be exploited to regulate expression of the therapy. Successful development of this approach requires engineering of gene switches and/or chimeric proteins which can sense and transduce the disease stimulus and effect the expression of a therapeutic molecule.

Although molecular switches have not yet been devised to achieve this goal, I envision the engineering of constructs that will be capable of regulating vascular hemodynamics where critical stenosis exists, enhancing nutrient transport to neurons, modulating the amplitude of intracellular calcium transients and preserving mitochondrion integrity. For example, the PAS gene family, many members of which are established signaling molecules mediating responses to environmental stimuli (reviewed in Ref. 11), could be co-opted to regulate pathophysiologic gene therapy. Specifically, the CNS response to hypoxia is known to involve the activation of HIF-1, transcription factor heterodimer that directs the expression of numerous protective genes which harbor specific cis hypoxia responsive elements (HREs; reviewed in Ref. 12). With this information one could develop gene constructs that would respond to cellular hypoxia, thus entraining the expression of a protective gene product to the pathophysiologic state. ${ }^{13,14}$ Whether this may have a therapeutic role in transient ischemia attack or chronic ischemic conditions remains to be determined.

Whereas this discussion was intended to raise awareness of potentially novel neurologic disease targets it did not address the many actively investigated gene therapy approaches for Parkinson's disease, brain tumors, Alzheimer's disease and spinal cord injury. Without doubt the mammalian CNS is a formidable target for any gene therapy. Most conspicuously this difficulty owes to the organs inaccessibility to noninvasive delivery methods. The acceptance of stereotactic neurosurgery as a companion to gene therapy will be necessary for this area to grow and reach its full therapeutic potential. The concomitant growth in the discovery of basic brain biologic mechanisms and refinements in gene delivery systems favor the development of novel gene therapy strategies. It is evident that definitive dissection of pathogenic mechanisms producing CNS disease is necessary to achieve the implementation of rational gene therapy. As the clinician is instructed by a thorough understanding of the particular patient and their disease, so shall the gene therapist be taught, guided and directed by the functional and dysfunctional states of the neural substrate. Through appreciation of the mechanisms and unique anatomical relationships defining the healthy and diseased brain will it be possible to develop and implement CNS gene therapy.

HJ Federoff

Aab Institute for Biomedical Sciences University of Rochester School of Medicine and Dentistry Rochester, NY, USA

\section{References}

1 McNamara JO. Emerging insights into the genesis of epilepsy. Nature 1999; 399 (Suppl): A15-A22.

2 New KC, Rabkin SD. GABA synthesis in astrocytes after infection with defective herpes simplex virus vectors expressing glutamic acid decarboxylase 65 or 67. J Neurochem 1998; 71: 23042312.

3 Robert JJ et al. Adenovirus-mediated transfer of a functional GAD gene into nerve cells: potential for the treatment of neurological diseases. Gene Therapy 1997; 4: 1237-1245.

4 Mody I, Schwartzkroin PA. Acute seizure models (intact animals). In: J Engel Jr, TA Pedley (eds). Epilepsy: A Comprehensive Textbook. Lippincott-Raven: Philadelphia, 1997, pp 397-496.

$5 \mathrm{O}^{\prime}$ Connor WM et al. Adenovirus vector-mediated gene transfer into human epileptogenic brain slices: prospects for gene therapy in epilepsy. Exp Neurol 1997; 148: 167-178.

6 Yenari MA et al. Gene therapy with HSP72 is neuroprotective in rat models of stroke and epilepsy. Ann Neurol 1998; 44: 584-591.

7 Freese A et al. Direct gene transfer into human epileptogenic hippocampal tissue with an adeno-associated virus vector: implications for a gene therapy approach to epilepsy. Epilepsia 1997; 38: 759-766.

8 Ahmed MM et al. Ionizing radiation-inducible apoptosis in the absence of p53 linked to transcription factor EGR-1. J Biol Chem 1997; 272: 33056-33061.

9 Advani SJ, Chmura SJ, Weichselbaum RR. Radiogenetic therapy: on the interaction of viral therapy and ionizing radiation for improving local control of tumors. Semin Oncol 1997; 24: 633638.

10 Aletich VA et al. Arteriovenous malformation nidus catheterization with hydrophilic wire and flow-directed catheter. Am J Neuroradiol 1997; 18: 929-935.

11 Taylor BL, Zhulin IB. PAS domains: internal sensors of oxygen, redox potential, and light. Microbiol Mol Biol Rev 1999; 63 479-506.

12 Semenza GL. Hypoxia-inducible factor 1 and the molecular physiology of oxygen homeostasis. J Lab Clin Med 1998; 131: 207-214.

13 Dachs GU et al. Targeting gene expression to hypoxic tumor cells. Nature Med 1997; 3: 515-520.

14 Dachs GU, Stratford IJ. The molecular response of mammalian cells to hypoxia and the potential for exploitation in cancer therapy. Br J Cancer 1996; 74 (Suppl 27): S126-S132. 